\title{
AGROSSISTEMAS - PERSPECTIVAS DE DESENVOLVIMENTO SUSTENTADO
}

\author{
Adriana Maria Nolasco ${ }^{1}$ \\ Alexandre Grimaldi de Castro ${ }^{2}$ \\ Edgar Marassi ${ }^{3}$
}

\section{RESUMO}

O conceito de desenvolvimento sustentado surgiu na última década como uma proposição alternativa a estratégia tradicional de desenvolvimento econômico e social. O setor agrícola não pode se furtar ao atendimento a estas novas prerrogativas, sendo assim fundamental o estabelecimento de bases consistentes de reflexão. A inserção deste novo conceito deve representar a possibilidade de aprimoramento técnico das atividades produtivas, sendo este fundamentado em procedimentos de negociação política entre os diversos atores sociais diretamente arrolados. $\mathrm{O}$ conhecimento científico

\section{CONCEITUAÇÃO INICIAL}

Diferentes formas e alternativas são encontradas em literatura para a definição de bases conceituais do desenvolvimento sustentado. Este tem sido citado de maneira cada vez mais frequente por diversos setores, sejam governamentais, empresariais ou acadêmicos, sem que no entanto sejam observadas iniciativas consistentes para normalização dos conceitos e idéias que permeiam a questão da sustentabilidade de sistemas naturais e antrópicos (LUBCHENCO et al., 1991).

Tecnicamente o conceito de sustentabilidade pode ser relacionado à capacidade de um sistema em propiciar meios de subsistência a determinada população em intervalos espaço/temporais definidos. Um sistema sustentável pode ser caracteriza- atual nos permite reconhecer referenciais, em especial quanto ao manejo de paisagens e inserção dos conceitos sócio-culturais e ambientais. Açōes de pesquisa voltadas à definição de diagnósticos ambientais e socioeconômicos apresentam-se como alternativas técnicas à elaboração de planos estratégicos de desenvolvimento regional, sendo fundamentadas por meio do reconhecimento de fatores condicionantes e condiçōes de contorno intrínsecas aos sistemas manejados e em prerrogativas de maior sustentabilidade da atividade agrícola nas dimensões espaço-temporais pertinentes.

do por apresentar um equilíbrio dinâmico - balanço/temporal - entre suas necessidades e os meios de sustentação necessários. Especificamente quanto ao setor agrícola, a implementação de um programa de desenvolvimento, sustentado está relacionado à gestão da base de recursos e à interação como os agentes sociais nos processos de produção, transformação e comercialização (SCHALLER, 1993).

O conceito de desenvolvimento sustentado pode ser analisado, ainda, quanto a seus aspectos

(1) Escola de Agricultura Luiz de Queiroz/USP - Piracicaba, São Paulo.

(2) Centro de Recursos Hídricos e Ecologia Aplicada, Escola de Engenharia de São Carlos/USP.

(3) Centro de Recursos Hídricos e Ecologia Aplicada, Escola de Engenharia de São Carlos/USP. 
ambiental (abordagem biofísica) e antrópico. 0 primeiro representa uma avaliação integrada dos processos produtivos e ambientais, das estratégias de manejo empregadas e dos procedimentos de conservação e utilização racional dos recursos naturais. O segundo trata especificamente dos componentes políticos, sociais e culturais, procurando-se a incorporação destes fatores na elaboração de planos de desenvolvimento sustentado. Objetiva a compreensão dos processos produtivos em escala mais ampla, com o resgate e conservação de valores culturais e ambientais em suporte a atividades específicas do setor:

Questões de cunho social assumem feição mais crítica quando analisadas economias e sociedades em desenvolvimento. Estas caracterizam-se por terem historicamente excluído determinados agentes e parcelas da sociedade durante processo de desenvolvimento regional. A implementação de Programas de Desenvolvimento Sustentado nestas regiões requer, assim, a integração de todos os elementos da sociedade diretamente afetados, de forma a conferir consistência política ao processo (MOTTA e MAY, 1994). O não envolvimento de uma determinada parcela da sociedade implica riscos acentuados a médio e longo prazos, tendo em vista o empobrecimento sócio-econômico e a possibilidade de agravamento substancial das crises políticas e sociais.

\section{AGROECOSSISTEMAS}

O conceito de uma agricultura alternativa surgiu como resultado de um processo de mobilização da opinião pública mundial na década de 80 em resposta aos riscos potenciais à saúde humana e qualidade ambiental reconhecidos nos sistemas de produção agrícola convencionais. O primeiro conceito, agricultura regenerativa, consistiu da proposição, algo intuitiva, de um sistema que refletisse menores gastos energéticos e de insumos químicos. A partir da incorporação de uma série de conceitos e fundamentos teóricos da Ecologia, fo- ram estabelecidas as bases da agroecologia, referencial teórico para o desenvolvimento dos agroecossistemas (ALTIERI, 1992). Ao contrário de sistemas convencionais de produção, o manejo de agroecossistemas prioriza a utilização de mecanismos naturais, em especial processos de interação intra e interespecíficos. O gerenciamento do balanço de massas (manutenção da fertilidade dos solos), p.e., é efetuado por meio da intensificação dos processos naturais de reciclagem de nutrientes, maximizando-se a participação de macro e microrganismos na decomposição e mineralização dos restos de colheitas e resíduos orgânicos derivados de outras atividades produtivas da propriedade rural. Procura-se minimizar ao máximo a entrada de insumos na forma de fertilizantes químicos e demais produtos halóctones. O controle fitossanitário é adotado por intermédio de técnicas de controle biológico, controle integrado de pragas e doenças e de forma mais avançada manejo integrado. Estes procedimentos incentivam, igualmente, a maximização dos processos naturais, baseando-se quase que exclusivamente na interferência sobre fatores de interação intra e interespecíficos.

\section{SISTEMAS PRODUTIVOS E MEIO AMBIENTE}

Grande parte dos sistemas de produção obedece a padrões estruturais e de manejo similares. São constituídos por plantações homogêneas, geralmente compostas por cultivares de alta produtividade. Os procedimentos de manejo apresentam intenso gasto de energia, sendo diretamente dependentes de matriz energética clássica, a qual pressupõe alta disponibilidade de recursos para manutenção dos sistemas de produção (HALL, 1990).

Os planos de manejo adotam, em sua maioria, uma abordagem reducionista quanto à questão ambiental, ficando restritos apenas aos aspectos agrícolas. A manutenção e maximização da produtividade são definidos como elementos centrais 
destes modelos de produção, sendo abordada em segundo plano a qualidade ambiental, a estabilidade sócio-econômica e a preservação de bens culturais e antropológicos (EDWARDS et al., 1993).

A avaliação de fatores ambientais extrínsecos ao manejo agrícola está voltada ao atendimento de exigências legais estabelecidas por órgãos governamentais. Mais recentemente, com uma maior mobilização da opinião pública nacional e internacional em torno da conservação ambiental, o setor tem-se deparado com crescentes pressões, muitas vezes refletidas em restrições de mercado (REIS, 1994). A análise e avaliação das externalidades derivadas do processo produtivo estão voltadas, no entanto, quase que exclusivamente para a sustentação dos níveis de produção, apresentando como objetivo principal a manutenção da capacidade competitiva frente a diferentes mercados. Externalidades negativas do ponto de vista da sociedade local/ regional não são comumente reconhecidas como de responsabilidade do setor produtivo, sendo transferidas para o âmbito governamental.

Embora a substituição de sistemas convencionais por agroecossistemas seja apresentada como proposta alternativa, esta parece, em princípio, tecnicamente inviável. As restrições observadas no processo produtivo são em grande parte resultantes das tecnologias agrícolas e industriais empregadas e do padrão de demanda (qualidade e características do produto) estabelecido pelo mercado. A complexidade envolvida na implementação e manejo de tais sistemas em escalas mais amplas resultaria em perda expressiva de competividade, em particular pelo aumento dos custos e queda imediata da produtividade por área plantada (HALL; HALL, 1993). Deve-se considerar, ademais, os ganhos efetivos desta ação frente aos riscos impostos ao setor e à sociedade a partir da retração desta atividade econômica.

Grande parte da comunidade científica ligada ao setor agrícola advoga que os sistemas convencionais são ainda passíveis de pequenos ajustes e correções, as quais podem possibilitar uma redu- ção significativa dos impactos ambientais sem que seja necessário uma ruptura do atual paradigma tecnicista. Em princípio, estes projetam pequenas melhorias quanto a qualidade ambiental quando comparados aos padrões obtidos por ajustes estruturais em sistemas convencionais, não justificando a implementação solada desta estratégia alternativas de manejo em programas conservacionais ou voltados ao desenvolvimento sustentado. A principal dificuldade concerne a necessidade de se estabelecer um rígido sistema de controle e a descapitalização de grande parte dos produtores rurais, os quais não apresentam condições econômicas ou técnicas favoráveis para proceder aos ajustes necessários.

\section{PRINCIPAIS IMPACTOS SOBRE O MEIO AMBIENTE}

A análise dos impactos ambientais de atividades agrícolas deve considerar a premissa de que ambientes distintos representam diferentes condições de contorno, implicando em graus variados de fragilidade e susceptibilidade a distúrbios naturais e antrópicos. A classificação quanto a amplitude e intensidade dos impactos mais frequientes passa a ser uma função do contexto ambiental onde se encontra implantado determinado empreendimento, bem como da escala de investigação.

Os principais impactos ambientais das atividades de produção agrícola, sobretudo intensivas, estão relacionados à redução da biodiversidade local/regional, mudanças climáticas, alterações dos ciclos hidrológicos e de nutrientes, mudanças nos fluxos de energia, aumento da entropia dos sistemas de paisagem e reflexos sobre a estrutura sócio-econômica e cultural. Como elementos secundários são indicados impactos sobre o solo, riscos de contaminação ambiental, descaracterização estética da paisagem e desertificação.

Os impactos mais expressivos sobre a biodiversidade são observados quando da substituição de 
ecossistemas naturais por plantações monoespecíficas. Permanecem incertos, todavia, os reflexos da redução da heterogeneidade estrutural em escala de paisagens sobre a biodiversidade, bem como o atual dimensionamento de áreas mínimas para preservação de parcela significativa da diversidade genética regional (ROSSITER, 1990). Uma série de incertezas permanece quanto a melhor estratégia de desenvolvimento de programas de preservação e sua compatibilização com as atividades produtivas. É necessário, todavia, a elucidação das mesmas para que as ações preservacionistas atinjam os objetivos esperados (ORIANS, 1990), sem que ocorra um comprometimento expressivo do setor produtivo.

A questão da biodiversidade é correntemente tratada como elemento exógeno ao processo produtivo, onde são estabelecidas áreas de preservação e conservação em que seja considerada de forma explícita sua relação com áreas de cultivo (ISHWARAN, 1992). A definição espacial das áreas de preservação segue orientação estabelecida pela legislação pertinente, na qual são definidas áreas não passíveis de exploração. Não há preocupação objetiva com a preservação do patrimônio genético das regiões sob influência direta e indireta desta atividade. A sistemática corrente corresponde a uma tentativa de maximização das áreas plantadas, tendo como referencial a oferta de produtos (commodities) e a redução dos custos imediatos de produção. Mesmo com a adoção de tecnologias de manejo agrícola e ambiental, observa-se uma tendência ao estabelecimento de abordagens reducionistas do problema.

Impacto relevante sobre o clima regional/global está relacionado, basicamente, à supressão de grandes extensões de florestas, em especial na região Amazônica e em áreas alagadas. Este processo dá-se comumente pelo emprego de queimadas, durante o qual são emitidas grandes quantidades de poluentes primários responsáveis pelo efeitoestufa (BOAG et al, 1994). A participação histórica do setor como agente deste processo é classificada como significativa. A inserção deste é observada também de forma indireta por meio da matriz energética, principalmente quanto aos sistemas intensivos de produção, onde são utilizadas quantidades expressivas de insumos e pelo consumo direto de combustiveis fósseis durante procedimentos de preparo dos solos, plantio e transporte. Com a degradação da qualidade ambiental, inclusive em áreas de cultivo, observa-se um aumento dos gastos energéticos, sobretudo em fertilizantes, para a manutenção dos níveis de produtividade. A anexação de áreas anteriormente consideradas como marginais colabora igualmente para o aumento no uso de insumos químicos, resultando em níveis mais elevados de entropia nos sistemas produtivos. Agroecossistemas, por não fazerem uso intensivo de insumos químicos e de certa forma contribuirem para a recuperação da fertilidade dos solos, apresentam relativa vantagem - a nível de sustentação funcional - sobre demais sistemas de cultivo.

Não obstante os recursos hídricos serem considerados como um dos principais. receptáculo de impactos advindos das atividades agrícolas (GISH; SADEGHI, 1993), observa-se uma ausência quase completa de esforços objetivos para implementação de programas específicos de gerenciamento deste recurso. Ao contrário do solo, que tem merecido crescentes esforços em pesquisa e desenvolvimento de novas tecnologia de manejo, os recursos hídricos são geralmente relegados às incertezas inerentes aos sistemas naturais. Os esforços e ações de manejo estão voltados, principalmente, a garantir o provimento de recursos para atividades específicas, como sistemas de irrigação, não sendo externada preocupação quanto ao dimensionamento e investigação da dinâmica espaço-temporal de estoques hídricos, bem como sua susceptibilidade a processos de contaminação (OBERLE; BURKART, 1994).

Impactos sobre a estrutura e dinâmica sócioeconômicas estão diretamente relacionados a uma tendência à concentração dos meioṣ de produção e mudanças nas relações de capital-trabalho, apresentando potencial de geração de conflitos relevante (BUTTEL, 1993). O êxodo em direção aos centros 
urbanos ocasiona uma relativa deterioração das condições de vida, aumento de problemas sanitários e de saúde pública e consequiente elevação dos custos indiretos de produção - externalidades negativas. Estes fatores são exacerbados pela ausência de infra-estrutura urbana condizente com o repentino crescimento populacional, e ainda descaracterização cultural dos agentes envolvidos.

O fator escala constitui, adicionalmente, elemento de extrema importância na avaliação e relativização dos impactos ambientais (RAFFY, 1994). A intensidade e importância relativa de determinada técnica de manejo sobre a estabilidade de processos funcionais é resultante, em parte, da escala de abordagem. Sistemas com maior amplitude espacial e potencial de dispersão tendem, em princípio, a exercer impactos mais significativos. Todavia, a avaliação destes impactos está diretamente relacionada à escala dos fenômenos analisados.

Da mesma forma, a implementação de processos de desenvolvimento sustentado deve ser avaliada segundo a escala analítica dos processos correlacionados. Os reflexos desta abordagem fazem-se mais claros através da adoção dos conceitos de manejo de paisagens ou de bacias hidrográficas. A dinâmica dos impactos observados em escala de microbacia, p. e., difere daquelas reconhecidas para bacias hidrográficas, demandando ações de manejo específicas.

A introdução de uma nova abordagem para o planejamento e manejo de sistemas agrícolas de produção - agroecossistemas, e seus reflexos sobre o meio ambiente, são igualmente uma função da escala de análise e observação. Impactos positivos mais imediatos fazem-se sentir a nível dos campos de cultivo, onde observa-se uma tendência ao estabelecimento de sistemas biologicamente mais estáveis. Sua influência em escalas mais amplas poderá ser observada de forma mais objetiva na medida em que outras medidas sejam adotadas, em particular quanto ao planejamento macro-ambiental. Nesta escala são analisadas as possibilidades de compatibilização das atividades produtivas com ações conservacionistas, em particular, quanto a manutenção da biodiversidade - biomas e ecossistemas particularmente ameaçados.

\section{MINIMIZAÇÃO DE IMPACTOS AMBIENTAIS}

Uma série de medidas intermediárias podem ser e estão em parte sendo adotadas para minimizar alguns impactos ambientais negativos, mesmo em se tratando de sistemas convencionais de produção. Investimentos na prevenção de processos de contaminação físico-química, comprometimento qualitativo dos recursos hídricos e atmosféricos, contenção de processos erosivos e de perda de solos e preservação de ecossistemas ameaçados constituem alguns exemplos (SHARPLEY; MEYER, 1994). A adoção do plantio direto por parte do setor, assim como desenvolvimento do conceito de sítios ambientais, representam ações que procuram adequar as atividades de produção a condicionantes previamente estabelecidas no ambiente.

Programas de educação ambiental constituem outro exemplo positivo. Uma série de conceitos são abordados durante a execução destes programas, muitas vezes com o envolvimento de comunidades locais. Estes atuam como instrumentos de difusão de novas perspectivas de avaliação do conceito de ambiente enquanto elemento integrante das atividades produtivas, colaborando no desenvolvimento de nova mentalidade entre produtores e sociedade local/regional.

Ademais, funciona como agente de interface social, permitindo o estabelecimento de espaço comum não convencional para interação direta dos diversos agentes e elementos representativos a nível local/regional.

Maior preocupação advém do fato de que grande parte das ações consideradas conservacionistas e de redução de impactos estar sendo adotada de maneira formal e sobretudo empírica. Fatores ambientais extrínsecos aos sistemas de produção são considerados estranhos ao processo produtivo. 
Segue-se paradigma clássico do ambiente como reservatório de recursos naturais. Na maioria dos casos, estas ações são adotadas por empresas agroindustriais, tendo como principais fatores condicionantes pressões de mercado e redução imediata dos custos de produção. A adoção de medidas conservacionistas por parte de pequenos e médios produtores faz-se, geralmente, por meio da utilização de tecnologias e sistemas tradicionais de manejo agrícola, nos quais alguns conceitos comuns a agroecologia são intuitivamente internalizados nos procedimentos de gestão das propriedades rurais.

\section{FATORES DE SUSTENTABILIDADE}

O estabelecimento de estratégias de desenvolvimento sustentado para o setor envolve uma mudança conceitual na acepção dos empreendimentos, sua relação espaço/temporal e a gestão coerente das externalidades positivas e negativas. Novas propostas devem estar aptas a lidar de forma objetiva com questões tão diversas quanto a biodiversidade, o desenvolvimento sócio-econômico regional e a qualidade ambiental (GROVE; EDWARDS, 1993).

Agroecossistemas tendem a apresentar uma estrutura mais flexível, sendo passíveis de ajustes e correções, de acordo com as variações intrínsecas às atividades produtivas. Na medida em que a sobrevivência do setor agrícola está, cada vez mais, relacionada com o potencial de adaptação a diferentes contextos (TIGRE, 1994), o surgimento de alternativas tecnológicas permite o estabelecimento de novos referenciais quanto a implementação de programas de desenvolvimento sustentado para o setor agrícola.

Considerando-se que um dos fundamentos do conceito de desenvolvimento sustentado é a gestão de recursos naturais, o estabelecimento dos níveis econômico e socialmente viáveis passa a ser definidos por fatores limitantes e balizadores intrínsecos do ambiente (OTZEN, 1993). Características físicas (abióticas) são genericamente consideradas como ele- mentos que definem de forma imediata a capacidade de suporte de determinado local. Em contraposição, condicionantes sócio-econômicos estabelecem o nível de tolerância às externalidades negativas geradas pelo setor produtivo. É fundamental, assim, compatibilizar a viabilidade técnica com o desenvolvimento sócio-econômico regional para que sejam alcançados os níveis de sustentabilidade desejados.

A probabilidade de sucesso de determinado empreendimento resulta da combinação de características intrínsecas do ambiente, ora denominadas condições de estado primitivas e dos meios tecnológicos empregados na alocação específica destes recursos. Este conceito diferencia-se da definição clássica de aptidão agrícola, visto ser necessário incorporar as dimensões temporal e social para que sejam alcançados níveis de sustentabilidade em intervalos mais longos. Assim, um local/região apresenta determinado nível de aptidão ao desenvolvimento de uma atividade produtiva/exploratória na medida em que conjuga uma série de fatores específicos, tais como solos, clima, relevo e recursos hídricos. Os níveis de sustentabilidade são definidos por estratégia de manejo que compatibilize a utilização dos recursos com a manutenção da integridade de mecanismos de auto-regulação (feedback) dos sistemas ambientais e a melhoria dos padrões de qualidade de vida das populações afetadas (UPRETI, 1994).

A possibilidade de serem estabelecidos sistemas produtivos sustentáveis por longos períodos é condicionada por processos fúncionais do ambiente em sua acepção mais ampla, na qual são objetivamente considerados fatores direta e indiretamente relacionados à atividade. 0 suprimento da demanda por determinado produto (commodity) não está mais circunscrito apenas à exploração dos recursos naturais em determinado intervalo espaço-temporal, mas também à compatibilização desta atividade setorial em um contexto que considere fatores extrínsecos ao seu manejo. O gerenciamento de recursos hídricos por meio do manejo de bacias hidrográficas é citado como um exemplo. A viabilidade operacional de diferentes setores e atividades 
produtivas é uma função da possibilidade de compatibilizar interesses e objetivos distintos, dinamicamente relacionados em um mesmo intervalo espacial - a bacia hidrográfica. Na medida em que determinada atividade implica em interferência ou competição pelo uso de determinado recurso, tal como exploração pesqueira ou atividades recreativas, a sustentabilidade ambiental da atividade produtiva passa a ser conceitual e operacionalmente questionada. FAETH (1993) indica que a consideração dos processos de degradação dos recursos naturais e interferência em outras atividades produtivas por meio de impactos difusos constituem parâmetros essenciais para avaliação da sustentabilidade econômica da agricultura, independentemente das técnicas de manejo empregadas.

A implementação do conceito de desenvolvimento sustentado pelo setor passa, ademais, por aspectos mais restritos às técnicas de manejo utilizadas. Determinadas estratégias de exploração dos recursos naturais podem resultar em fatores restritivos ou limitantes à própria atividade fim (OTZEN, 1993). O manejo de agroecossistemas, através da incorporação de nutrientes por intermédio do aproveitamento de resíduos de colheitas, bem como a proteção dos recursos hídricos e edáficos por técnicas conservacionistas, proporcionam maior sustentabilidade à atividade exploratória, na medida em que processos funcionais intrínsecos ao ambiente são incorporados às técnicas de manejo (HALL; HALL, 1993).

A diferenciação conceitual de um novo modelo de desenvolvimento econômico e de produção agrícola reporta-se, desta forma, à incorporação explícita de valores e prerrogativas ambientais aos procedimentos de manejo da base de recursos naturais. Neste caso, passa-se a tratar de manejo ambiental ou de agroecossistemas, dentro do qual insere-se, inclusive, a manutenção das atividades de produção. Inverte-se a sistemática atual, na qual o campo de cultivo é considerado como elemento extrínseco ao ambiente e isento de quaisquer processos interativos intra e inter-sistemas. Estes pas- sam a ser operacionalmente inseridos em um contexto mais amplo, no qual a produção agrícola constitui um dos elementos de gerenciamento e gestão do ambiente. Neste particular, a introdução dos conceitos de agroecossistemas ou da agroecologia vem fundamentar tais práticas, na medida em que são conceitualmente concebidos com base em processos interativos, incorporando diretamente a noção de sinergia inerente aos ecossistemas naturais.

A extensão do ambiente por intermédio do conceito de manejo de agroecossistemas propicia, assim, elementos teórico-operacionais para a abordagem de desafios atualmente contrapostos ao desenvolvimento agrícola. Permite a manutenção da biodiversidade e qualidade ambiental, os níveis de produtividade e atenção às necessidades de sustentação de sociedades regionais. A conservação ambiental, em particular preservação da biodiversidade, é tratada em escala não apenas pontual, mas por meio da conjugação dos diferentes elementos - ecossistemas - componentes de um ambiente. Tal abordagem possibilita inserir de forma objetiva aspectos anteriormente relegados a segundo plano, em especial aqueles relacionados a interdependência entre processos funcionais e padrões estruturais do ambiente.

O papel do reordenamento espacial de paisagens no desenvolvimento sustentado representa à diluição, no espaço e no tempo, das diversas externalidades geradas por atividades e interesses setoriais. Sistemas heterogêneos permitem o estabelecimento de bases mais estáveis de produção e desenvolvimento, na medida em que possibilitam a coexistência de diferentes prerrogativas produtivas e preservacionistas em um mesmo espaço e a conjugação de diferentes estratégias de desenvolvimento na dimensão temporal (SELMAN, 1992).

A gestão de um novo modelo de produção pode ser resumida como a internalização de conceitos ambientais mais amplos no gerenciamento de uma atividade específica. A produção passa a ser integrada em um plano de gestão ambiental que considere de forma objetiva as prerrogativas de de- 
senvolvimento multissetorial e a preservação de patrimônio ambiental e cultural inerentes a determinado local e/ou região.

\section{ESTRATÉGIAS DE TRANSIÇÃO E IMPLEMENTAÇÃO}

A adoção de uma sistemática de sustentabilidade do desenvolvimento econômico e social requer o estabelecimento de estratégias de transição, voltadas à transposição, o menos traumática possível, do modelo atual para o sistema de gerenciamento pretendido. Os fatores que balizam tal ação estão relacionados ao reconhecimento das condições de estado do ambiente, de táticas específicas conforme análise de riscos ambientais/econômicofinanceiros e treinamento/conscientização dos agentes sociais diretamente participantes.

A meta não constitui a substituição de um sistema estanque por outro. Ao contrário, representa uma mudança em especial cultural na administração dos processos envolvidos. Passa-se a trabalhar através do reconhecimento de desconformidades e da implementação de procedimento de ajustes de forma dinâmica, onde o papel dos sistemas de retroalimentação são fundamentais para que sejam alcançados níveis aceitáveis de qualidade e estabilidade do processo produtivo. De forma similar aos sistemas de auto-regulação de fenômenos naturais, o conceito de cibernética induz ao estabelecimento de uma nova dinâmica de gerenciamento, baseada e resultante de processos e mecanismos de retroalimentação - feedbacks.

De forma resumida, elencam-se as etapas de planejamento e gerenciamento como instrumentos básicos em programas de gestão ambiental e desenvolvimento sustentado. Um planejamento estratégico, elaborado de acordo com diagnóstico e prognósticos agro-ambientais, define a direção e sistemática a serem seguidas na operacionalização de processos de transição para o desenvolvimento sustentado. Ao gerenciamento ambiental cabe a manutenção e ajuste dos sistemas de manejo implementados, utilizando-se das ferramentas de monitoramento para acompanhamento de fenômenos e processos relevantes às atividades produtivas.

Instrumentos de Planejamento e Gerenciamento Ambiental - O primeiro passo consiste no reconhecimento detalhado dos fatores diretamente relacionados ao processo a ser gerenciado e aos níveis de inter-relação existentes. No caso de sistemas ambientais, deve-se considerar, em especial, a dinâmica espaço-temporal de determinados fenômenos, bem como os níveis de incerteza inerente ao conhecimento científico-tecnológico atual. A gestão de sistemas com alto índice de incerteza exige a adoção de uma estratégia mais prudente, na qual as condições de contorno estabelecidas para gerenciamento comportem uma maior margem de segurança (BARBERA et al., 1992).

O reconhecimento das relações espaciais entre os diversos elementos de uma paisagem pode ser relacionado como a primeira internalidade a ser objetivamente analisada. Estas são inicialmente circunstanciadas através da elaboração de zoneamentos ambientais, em especial daqueles denominados ecológico-econômicos. Este instrumento analítico permite estabelecer ao grupo de gestão uma visão sinóptica das potencialidades de um sistema ambiente e o reconhecimento das inter-relações entre os diversos componentes de uma paisagem (HABER, 1990). Tais informações são de fundamental importância quando consideradas a inserção e interrelação de diferentes setores e processos produtivos dentro de uma mesma unidade geoambiental.

Este procedimento pode ser adotado em diferentes escalas espaço-temporais. Projeta-se o zoneamento de uma bacia hidrográfica ou microrregião administrativa da mesma forma como é efetuada a compartimentação por unidades geoambientais em locais direcionados à produção agrícola. Em ambos os casos são previamente estabelecidas e consensadas normas e princípios que permitem a compartimentação ambiental em zonas homogêneas. 
O estabelecimento de uma relação entre padrões estruturais de paisagens e processos funcionais - mais afeitos às estratégias de manejo - permite a simulação do comportamento dinâmico de determinado componente frente à adoção de novas tecnologias de manejo. É possível projetar o comportamento provável de determinada classe de recursos, p.e. hídricos, frente a implantação, expansão ou redirecionamento das sistemáticas de manejo (SUGIHARTO et al., 1994). Através destes prognósticos detectam-se previamente possíveir conflitos, permitindo a proposição de estratégias alternativas de gerenciamento e exploração.

A análise de processos históricos de uso/ocupação de determinada região, bem como a evolução na adoção de diferentes tecnologias de manejo agrícola e ambiental, permite o estabelecimento de diagnósticos ambientais (TURNER, 1990). A partir destes, são definidas estratégias de ação que contemplem intervenções de curto, mẹ́dio e longo prazos, baseadas nas principais tendências e limitações observadas quanto as diferentes processos de desenvolvimento reconhecidos em determinada região.

A inserção de conceitos ambientais ao planejamento estratégico está intimamente relacionada à disposição de informações que permitam o reconhecimento imediato das condições de estado dos sistemas manejados (diagnósticos ambientais), bem como projeção dinâmica dos fenômenos mais relevantes (prognósticos). A seguir, são elaboradas e detalhadas ações específicas de manejo, voltadas à correção e otimização dos processos produtivos, de forma a torná-los mais sustentáveis.

Monitoramento Ambiental - 0 estabelecimento de sistemas de monitoramento ambiental favorecem igualmente o controle dos processos envolvidos em atividades produtivas. Estas técnicas podem ser classificadas de acordo com os fenômenos observados ou ainda por estarem focadas a estrutura ou funcionamento do ambiente. Técnicas de monitoramento estrutural estão relacionadas à observação das variações espaciais da cobertura vege- tal, qualidade das águas e controle atmosférico. Processos funcionais referem-se em particular às taxas de produção, interação e inter-relação entre os diversos componentes do ambiente.

A análise destes dados, através de técnicas de modelagem matemática, permite a simulação dos possíveis cenários advindos de determinada modificação estrutural do ambiente - projetada dentro do programa de manejo ou por eventos catastróficos. $\mathrm{O}$ planejamento e implantação de agroecossistemas em determinada área de uma microbacia ou bacia hidrográfica, com retirada de parte da cobertura vegetal nativa, permite antever o aumento da carga de escoamento dos tributários e rios principais, bem como as possíveis variações quanto à qualidade de água (GALLARD et al, 1994). Uma outra aplicação trata da avaliação do balanço de massas relativo à utilização de fertilizantes e técnicas de manejo. A partir da caracterização de parâmetros-chave, tais como recobrimento do solo e uso de fertilizantes, é possível avaliar as taxas de transporte e fixação de nutrientes nas parcelas manejadas, antevencio-se possíveis reflexos sobre os demais elementos componentes da paisagem (ou bacia hidrográfica), bem como a eficácia das técnicas empregadas frente aos resultados projetados e alcançados tanto a nível de produção quanto em qualidade ambiental (YAKOWITZ et al., 1993).

O monitoramento de parâmetros hidrológi$\cos$, inclusive meteorológicos, aliado a uma avaliação de características estruturais dos solos e lençol subsuperficial, permite estabelecer um programa de gerenciamento de recursos hídricos (ULERY et al., 1993). A avaliação criteriosa da disponibilidade e qualidade dos recursos, ademais do favorecimento na resolução de conflitos de uso múltiplo, permite um manejo sustentado das atividades. Como um dos fatores essenciais ao estabelecimento de taxas ótimas de produção, este representa um dos elementos básicos de monitoramento em planos de gestão ambiental. A utilização de modelos agrometeorológicos, por outro lado, permite estabelecer sistemas de previsão de safras, nos quais estão 
inclusas projeções de mobilização de recursos naturais por parte dos agrossistemas (RUNNING et al., 1989).

Alguns parâmetros funcionais são igualmente úteis para a avaliação da eficácia dos programas de transição e gestão do desenvolvimento sustentado. $O$ reconhecimento de bioindicadores apresenta-se como uma das técnicas passíveis de utilização. Através do acompanhamento da dinâmica populacional - expressa muitas vezes pela abundância ou frequiência relativas de determinadas espécies - é possível estimar o grau de estabilidade de um sistema ambiente. O surgimento de espécies oportunistas, p.e., é geralmente considerada como um indicativo de estresse ambiental. Índices de respiração edáfica, assim como taxas de decomposição, são igualmente utilizados para a avaliação da estabilidade ou estresse de determinado ambiente.

Parâmetros sócio-econômicos constituem elementos de monitoramento que permitem identificar as externalidades intrínsecas a agricultura. Indicadores demográficos, econômicos e sociais possibilitam a detecção e acompanhamento de conflitos e desconformidades derivados da implantação de sistemas convencionais e introdução de sistemas alternativos de produção agrícola. As informações obtidas através do monitoramento sócio-econômico possibilitam a inserção das dimensões sociais no planejamento e gerenciamento de programas de desenvolvimento sustentado. Na medida em que uma das prerrogativas da sustentabilidade trata especificamente da qualidade de vida das populações locais/regionais, métodos de monitoramento sócioeconômicos constituem instrumentos importantes $\mathrm{n} \exists$ avaliação e acompanhamento de processos de transição para o desenvolvimento sustentado.

Acompanhamento Macro-Ambiental - O sucesso na implementação de programas de desenvolvimento sustentado está diretamente relacionado à gestão dos diversos processos envolvidos. Cabe neste caso, o estabelecimento de ferramentas analítico-operacionais que permitam o constante acompanhamento e diagnóstico dos programas de tran- sição e gestão. Instrumentos tais como auditorias ambientais, bem como programas de difusão tecnológica e educação ambiental constituem exemplos já testados em outros contextos e que alcançaram relativo sucesso.

A exemplo de programas de qualidade total, o sucesso da implementação de procedimentos de gestão ambiental está relacionado ao grau de comprometimento da cada um dos agentes envolvidos no gerenciamento do processo produtivo. Cabe, entretanto, uma discussão mais ampla quanto à adequacidade dos referenciais técnicos estabelecidos. É necessário, conforme discutido acima, considerar as peculiaridades de cada região analisada, Não parecendo coerente, em primeira instância, o estabelecimento de regras genéricas. A simples transposição de valores estabelecidos como referenciais para outras regiões pode resultar em efeito contrário ao esperado.

Ao invés de incentivar a implantação de programas de desenvolvimento sustentado, estes serviriam apenas como entraves, restringindo a abordagem da proposta original de sustentabilidade.

Uma série de incentivos à adoção de programas de desenvolvimento sustentado por parte do setor pode ser estabelecida. Programas de incentivos e subsídios governamentais, política agrícola e agrária, estabelecimento de sistemas mais estáveis para definição de preços e exploração de nichos específicos de mercado são alguns dos fatores atualmente considerados comó prioritários para o estabelecimento de sistemas sustentáveis de produção (ZHAO et al., 1991). Embora os sistemas convencionais de produção agrícola sejam considerados como inviáveis a nível macroeconômico (HALL; HALL, 1993), a manutenção de agroecossistemas apresenta-se como igualmente impraticável em níveis economicamente aceitáveis, visto estes não apresentarem padrões de competitividade compatíveis com os atuais referenciais de mercado. Desta forma, a viabilização do setor, seja qual for a tecnologia ou sistema de produção adotados, passa por uma redefinição das políticas agrícola e de preços. A difusão de 
agroecossistemas requer, ademais, o redirecionamento e mudanças das atuais estruturas de ensino e pesquisa, de forma a se estabelecer bases mais consistentes de suporte ao desenvolvimento do setor por períodos mais longos.

\section{DISCUSSÃO PRELIMINAR}

Os principais desafios à adoção de uma estratégia de desenvolvimento sustentado estão relacionados, em primeira instância, a aspectos políticos e conceituais. A mudança no atual posicionamento político quanto ao desenvolvimento econômico e social e sua associação com a crise ambiental emergente representa força propulsora para futuros desenvolvimentos técnico-científicos. Todavia, o aparato científico-tecnológico não está capacitado a definir sozinho os direcionamentos necessários à adoção de um programa genérico ou setorizado de desenvolvimento sustentado.

Observa-se uma necessidade premente por mudança conceitual/operacional do atual modelo de produção, na medida em que o mesmo não oferece perspectivas satisfatórias de sustentabilidade por períodos mais longos. É preciso, todavia, reconhecer os níveis de risco aceitáveis para que as mudanças necessárias sejam implementadas sem incorrer em comprometimento significativo dos interesses e investimentos estabelecidos. Um sistema de seguridade deve ser definido de forma a garantir, sob determinados critérios e metas acordados, tanto a perenidade e estabilidade ambiental como a do próprio setor produtivo. A retração ou inviabilização econômica deste setor representam impactos sociais e ambientais ainda mais acentuados do que a manutenção das atuais normas de produção.

A resolução de questões colocadas como eminentemente técnicas, tais como impactos ambientais no âmbito regional, globalização econômica e de interesses setoriais, dentre outras, está vinculada, em primeira instância, ao posicionamento político dos diversos segmentos da sociedade. Es- perar mudanças do consciente coletivo para que os aprimoramentos necessários façam-se presentes é impossível em função da premência e incerteza quanto aos aspectos temporais de fenômenos emergentes. Resta aos formadores de opinião, oriundos dos mais diversos setores, sejam governamentais, civis, científicos ou empresariais, a predisposição ao estabelecimento de bases consistentes de negociação, com a proposição de ações concretas e de uma sistemática de interação intensiva.

A manutenção de alguns paradigmas é incompatível com o estabelecimento de nova sistemática de manutenção e desenvolvimento das sociedades modernas. É difícil imaginar a sustentabilidade físico-orgânica da sociedade mantendo-se como padrões as atuais referências de demanda por bens e serviços. A transposição dos padrões de consumo de sociedades desenvolvidas para países em desenvolvimento é fisicamente impossível. Por outro lado, a manutenção dos atuais níveis de desigualdade social tem implicado distorções de difícil sustentação, com consequiências imprevisíveis quanto à estabilidade e perenidade dos atuais sistemas de organização social e política.

A ruptura de paradigmas é representada pela adoção de estratégias não-imediatistas e implementação de ações integradas de desenvolvimento, com os interesses setoriais tratados dentro de uma perspectiva mais ampla de sustentabilidade de toda a sociedade. A premência por mudanças concretas e emergenciais remete à articulação de estratégias orientadas por um posicionamento político, sustentado por disposições sociais e científicas arregimentadas em um mesmo fim: sobrevivência da sociedade humana sobre fundamentos de maior sustentabilidade.

Os conceitos de agroecologia, agrossistemas e gestão ambiental insere, neste contexto, conceito semelhante à re-engenharia dos processos de produção. A abordagem pretendida, de caráter matricial, tende a integrar as diversas atividades do setor em procedimentos de gerenciamento de processos. Neste caso, os processos são representados não somente pelo sistema produtivo em sua acepção mais restri- 
ta, como também pelos fenômenos ou processos ambientais relacionados. O setor agrícola assume uma postura não apenas de consumidor, mas gestor ambiental também das externalidades derivadas do uso dos recursos naturais. Na medida em que outras atividades relacionadas ao processo produtivo dependem da utilização intensiva de determinado recurso, novas perspectivas são adicionadas ao processo de gestão. Administra-se, p. e., o ciclo ou balanço hidrológico em escala regional, atendo-se não somente ao suprimento da demanda imediata e setorial, mas também a administração temporal e interativa do recurso. A adoção de procedimentos de co-responsabilidade por parte de setores representativos da sociedade constitui fator imperativo ao sucesso de programas de desenvolvimento sustentado.

As externalidades positivas e negativas devem ser negociadas em fórum mais amplo, de forma a adequar os níveis de disposição espaço-temporal à capacidade de absorção e mudanças das sociedades afetadas. $\mathrm{Na}$ medida em que estes não são considerados como sistemas fechados, as ex- ternalidades geradas pelo setor são sentidas em diversos segmentos da sociedade, ocasionando, por outro lado, reflexos e ações positivas e negativas sobre o próprio setor.

Independentemente dos avanços científicos/ tecnológicos observados e projetados, o elemento social continuará indubitavelmente a ser fundamental e participativo de qualquer atividade produtiva. Na medida em que se considere a existência e importância de sistemas cibernéticos (retroalimentação) na natureza, inclusive humana, as condições atualmente predispostas definirão os cenários futuros.

Neste contexto a inserção de tecnologias ou abordagens teórico-conceituais alternativas representam instrumentos para gerenciamento ou gestão dos processos envolvidos. A diferenciação de diretrizes quanto ao desenvolvimento sustentado permeia níveis mais abstratos, eminentemente relacionados às interações e posicionamentos políticos por parte dos diferentes setores e agentes representativos da sociedade.

\section{BIBLIOGRAFIA}

ALTIERI, M.A. Sustainable agricultural development in Latin America: exploring the possibilities. Agriculture, Ecosystems \& Environment. V. 39 (1-2), p. 1-19, 1992.

BARBERA, G. et al. Diffusion of innovative agricultural production systems for sustainable development of small islands: a methodological approach based on the science of complexity. Environmental Management. V. 16(5), p. 667-79. 1992.

$\mathrm{BOAG}$, S. et al. Monitoring and reducing greenhouse gas emission from agriculture, forestry and other human activities. Climatic Change. V. 27(1), p. 5-12. 1994.

BUTTEL, F.H. The sociology of agricultural sustainability: some observations on the future of sustainable agriculture. Agriculture, Ecosystems and Environment. V. 46, p. 175-86. 1993.

EDWARDS, C.A; GROVE, T.L.; HARWOOD, R.R.; COLFER, C.J.P. The role of agroecology and integrated farming systems in agricultural sustainability. Agriculture, Ecosystems and Environment, v. 46, p. 99-121. 1993.
FAETH, P. An economic framework for evaluating agricultural policy and the sustainability of production systems. Agriculture, Ecosystems and Environment. V. 46, p. 161-73. 1993.

GALLART, F; LLORENS, P.; LATRON, J. Studying the role of old agricultural terraces on runoff generation in a small Mediterranean mountainous basin. Journal of Hydrology. V. 159(1-4), p. 291-303, 1994.

GISH, T.J.; SADEGHI, A. Agricultural wate quality priorities: a symposium overview. Journal of Environmental Quality.v. 22(3), p. 389-91. 1993.

GROVE, T.L.; EDWARDS, C.A. Do we need a new developmental paradigm? Agriculture, Ecosystems and Environment. V. 46, p. 135-45. 1993.

HABER, W. Basic concepts of landscape ecology and their application in land management. Physiology and Ecology of Japan. V. 27, p. 131-46. 1990. 
HALL, C.A.S. Sanctioning resource depletion: economic development and neo-classical economics. Ecologist. V. 20, 31-66. 1990.

HALL, C.A.S.; HALL, M.H.P. The efficiency of land and energy use in tropical economies and agriculture. Agriculture, Ecosystems and Environment. V. 46, 1-30. 1993.

ISHWARAN, N. Biodiversity, protected areas and sustainable development. Natural Resources. V. 28(1), p. 18-25. 1992.

LUBCHENCO, J. et al. The sustainable biosphere initiative: an ecological research agenda, Ecology. V. 72 (2), p. 371-412. 1991.

MOTTA, R. S.; MAY, P.H. Valorando a Natureza-Análise Econônica para o Desenvolvimento Sustentável. Rio de Janeiro, Ed. Campos, $1994,195 \mathrm{pp}$.

OBERLE, S.L.; BURKART, M.R. Water reource implications of Midwest agroecosytems. Journal of Environmental Quality. v. 23, p. 4-8, 1994.

ORIANS, G.H. Ecology and conservation biology: mutually supprtive sciences. Physiologyand Ecologyof Japan. V. 27, p. 151-65. 1990.

OTZEN, U. Reflections on the principles of sustainable agricultural development. Environmental Conservation. v. 20(4), p. 31016.1993 .

RAFFY, M. Change of scale theory - a capital challenge for space observation of Earth Introduction. International Jounal of Remote Sensing: v. 15(12), p. 2353-58. 1994.

REIS, M.J.L. ISSO 14.000 - Riscos da certificação ambiental. Agroanálises. v. 14(2), 48. 1994.

ROSSITER, A. Ecology, environment and economics: A pandoran perspective. Physiologyand Ecology of Japan. v. 27, p. 169-89. 1990.

RUNNING, S.W. et al. Mapping regional forest evapotranspiration and photosynthesis by coupling satellite data with ecosystem simulation. Ecology. v. 70, p. 1090-1101.1989
SCHALLER, N. The concept of agricultural sustainability. Agriculture, Ecosystems and Environment. V. 46, p. 89-97. 1993.

SELMAN, P. Na investigation of the potencial for landscape ecology to act as a basis for rural land plans: Jounal Environmental Management. V. 35: 281-99. 1992.

SHARPLEY, A.; MEYER, M. Minimizing agricultural nonpoint-source impacts: a symposium overview. Journal Environmental Quality. V. 23(1), p. 1-3. 1994.

SUGIHARTO, T.; MCINTOSH, T.H.; UHRIG, R.C.; LARDINOIS, J.J. Modeling alternatives to reduce dairy farm and watershed nonpoint source pollution. Journal of Enviromental Quality.v. 23(1), p. 18-24. 1994.

TIGRE, P. V. Techologia e meio ambiente - oportunidades para a indistria. RJ, Ed.UFRJ, 1994. 139 pp.

TURNER, M.G. Landscape changes in nine rural counties in Georgia. Photogrammetric Engiheering and Remote Sensing. v. 56(3), p. 379-86.1990.

ULERY, R.L.; VAN METRE, P.C.; CROSSFIELD, A . S. Trinity river basin, Texas. Water Resources Bulletin. V. 29(4), p. 685-711. 1993.

UPRETI, G. Environmental conservation and sustainable development require a new development approach. Environmental Conservation. v. 21(1), p. 18-29. 1994.

ZHAO, F; HITZHUNSEN, F; CHERN, W. Impact and implications of price policy and land degradation on agricultural growth in developing coutries. Agricultural Economics. v. 5, p. 311-24. 1991.

YAKOWITZ, D.S. et al. Decision support system for evaluating the effects of alternative farm management systems on water quality and economics. Water Science and technology. V. 28(3-5), p. 47-54. 1993.

\begin{abstract}
The concept of sustainable development appears at the decade as na alternative to the tradictional strategies of economic and social development. The agricultural sector could not ignore thes new statements in establishing consistent bases of reflextion. A new concept must represent the possibility of technical improvement in this productivy activities, based at political negotiation among diffrent society sectors. The present scientific knowledge allow us to recognize references, specially concerning land-

scape management and socio-cultural and environmental issues. Research actions concerning environmental and socioeconomic prognosis and diagnosis represent technical alternatives to strategic planning programs for regional development. These actions are based on the recognition of condictioning factors and contour condictions intrinsec of managed environments and a sustainability prerrogatives for agricultural activities at proper space and temporal scales.
\end{abstract}

